\title{
Stellar Abundances: The r-Process and Supernovae
}

\author{
JOHN J. COWAN \\ Department of Physics and Astronomy, University of Oklahoma \\ Norman, OK 73019, USA \\ CHRISTOPHER SNEDEN \\ Department of Astronomy and McDonald Observatory, University of Texas \\ Austin, TX 78712, USA
}

Stellar abundance observations are providing important clues about the relationship between supernovae (SNe) and the rapid neutron capture process (i.e., the r-process). Although the site for the r-process is still not identified, events in and around SNe have long been suspected. Abundances of heavy neutron-capture elements in a number of stars suggest a robust r-process operating over billions of years, constraining astrophysical and nuclear conditions in supernova models. Variations in lighter n-capture element abundances - observed only very recently in any stars - could be explained as a signature of certain supernova models, or might require multiple r-process sites with different mass ranges or frequencies of SNe. Recent observations of elemental abundance scatter in the early Galaxy are consistent with earlier suggestions of a restricted range of SNe responsible for the r-process.

\subsection{Introduction}

The elements heavier than iron are synthesized in neutron processes, either in the (s)-low or (r)-apid process. In the $s$-process the timescale for neutron capture $\left(\tau_{n}\right)$ is much longer than the electron (beta)-decay $\left(\tau_{\beta}\right)$ timescale. For the $r$-process, however, $\tau_{n}<<\tau_{\beta}$ with many neutrons captured in a very short time period. As a result, neutron captures proceed into very neutronrich regions far from the stable nuclei, where very little experimental nuclear data is available. This element synthesis is intimately connected to the late stages of stellar evolution, with the $s$-process occurring in the thermally pulsing helium shells of asymptotic giant branch (AGB) stars of low- and intermediate-mass $\left(\mathrm{M} \sim 0.8-8 \mathrm{M}_{\odot}\right)$ (see, e.g., the review by Busso, Gallino, \& Wasserburg 1999). Supernovae have long been suspected as the site for the $r$-process (see recent reviews by Truran et al. 2002; Sneden \& Cowan 2003; Cowan \& Sneden 2003). Recent abundance observations in metal-poor 
(i.e., low iron fractions) Galactic halo stars are providing new insights into these synthesis processes.

\subsection{Abundance observations}

A number of studies spanning decades have examined $n$-capture abundances in metal-poor $([\mathrm{Fe} / \mathrm{H}]<-1)$ Galactic halo stars (see e.g., Spite \& Spite 1978; Gilroy et al. 1988; McWilliam et al. 1995; Ryan, Norris, \& Beers 1996; Sneden et al. 1996; Burris et al. 2000; Johnson \& Bolte 2001; Hill et al. 2002). We show in Figure 1.1 the abundances of the heaviest $n$-capture elements (Ba and above, $\mathrm{Z} \geq 56)$ in four such stars: CS 22892-052 $([\mathrm{Fe} / \mathrm{H}]$ $=-3.1$, Sneden et al. 2003), HD $115444([\mathrm{Fe} / \mathrm{H}]=-3.0$, Westin et al. 2000), $\mathrm{BD}+17^{\circ} 3248([\mathrm{Fe} / \mathrm{H}]=-2.1$, Cowan et al. 2002) and CS 31082-001 $([\mathrm{Fe} / \mathrm{H}]$ $=-2.9$, Hill et al. 2002). The abundances for CS 22892-052 are absolute, while the others have been vertically displaced for display purposes. These stellar elemental abundances are compared with the solar system $r$-process abundances (solid lines in Figure 1.1) that have been scaled (i.e., shifted downward to compensate for the difference in metallicities) to match the observed ( $r$-process element) Eu. The $r$-process elemental abundances were determined from deconvolving the solar system abundances into their two components, and employed the "classical" model to obtain the individual $s$-process contributions (see Burris et al. 2000).

The comparisons indicate that there is an excellent overall agreement from Ba through the $3^{\text {rd }} r$-process peak between these metal-poor $n$-capture-rich stars and the scaled (or relative) solar system $r$-process abundances. These comparisons suggest further that early in the history of the Galaxy most (all?) of the $n$-capture elements observed in these stars were synthesized by the $r$-process, even those such as Ba that are formed in the $s$-process in solar system matter. These elements formed early in the Galaxy could not have been synthesized by low-mass stars, the primary sites for the $s$ process, with long evolutionary time scales. The detections of the $r$-process elements in these old halo stars, in fact, suggest that the $r$-process sites in the earliest stellar generations, the progenitors of the halo stars, were rapidly evolving. The agreement between the solar system abundances and the elemental abundances in the halo stars, for at these $r$-process rich stars, also puts strong constraints on the conditions in, and suggests a robust nature of, the $r$-process. 


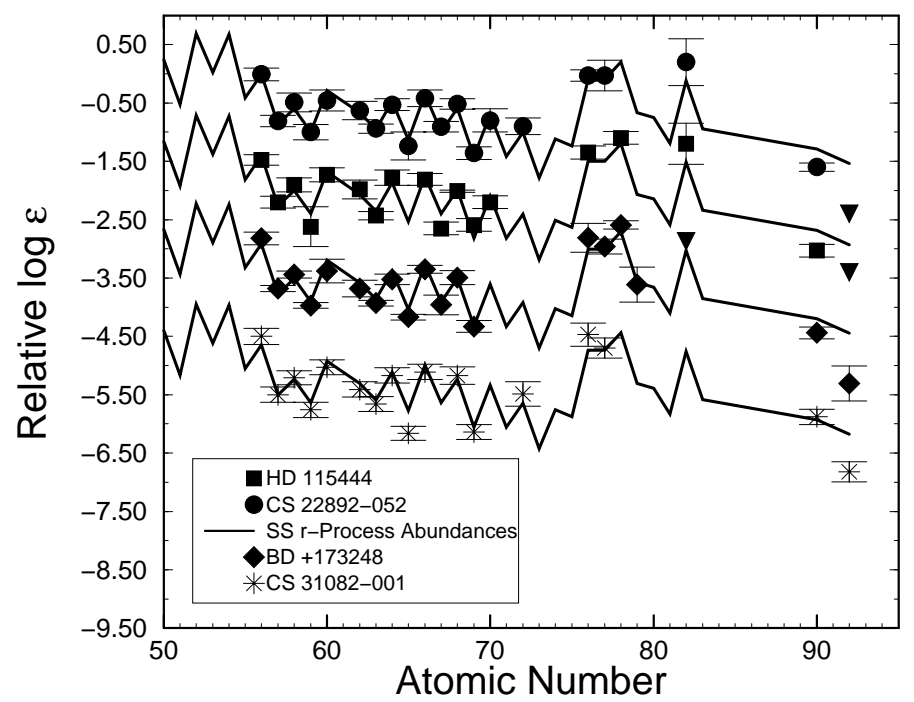

Fig. 1.1. A comparison of of the scaled solar system $r$-process abundances (solid line) with the $n$-capture element abundances in the the four Galactic halo, metalpoor stars CS 22892-052, HD 155444, BD +17 3248 , and CS 31082-001 (see Westin et al. 2000; Cowan et al. 2002; Hill et al. 2002; Sneden et al. 2003). The abundances for CS 22892-052 are absolute, while the other abundances have been vertically shifted for display purposes. Upper limits are indicated by inverted triangles. $\left(\log \epsilon(\mathrm{A}) \equiv \log _{10}\left(\mathrm{~N}_{\mathrm{A}} / \mathrm{N}_{\mathrm{H}}\right)+12.0\right.$, for elements A and B.) (After Cowan \& Sneden 2003.)

\subsection{The site or sites for the r-process}

We can obtain additional clues about the nature of the $r$-process and the possible astrophysical sites by including the light $n$-capture elements in our abundance analyses. Only recently have there been detections of a significant number of these light elements in the metal-poor stars. We show in Figure 1.2 the most recent observational data for the star CS 22892-052, including detections and upper limits for $13 n$-capture elements below $\mathrm{Ba}$, from Sneden et al. (2003). The agreement between the solar system curve and the heavier elements $(Z>56)$ illustrated in Figure 1.1 does not seem to extend to the lighter elements. While $\mathrm{Ru}, \mathrm{Nb}$ and perhaps $\mathrm{Rh}$ are consistent, other notable elements such as $\mathrm{Ag}, \mathrm{Pd}$ and Mo fall noticeably below the same curve that matches the abundances of the heavier $n$-capture elements. We note that while this star has the most complete data set for these lighter elements, other metal-poor stars show a similar pattern.

The abundance comparisons shown in Figure 1.2, including the new lighter element data, could be explained in several manners. One possibility is that 


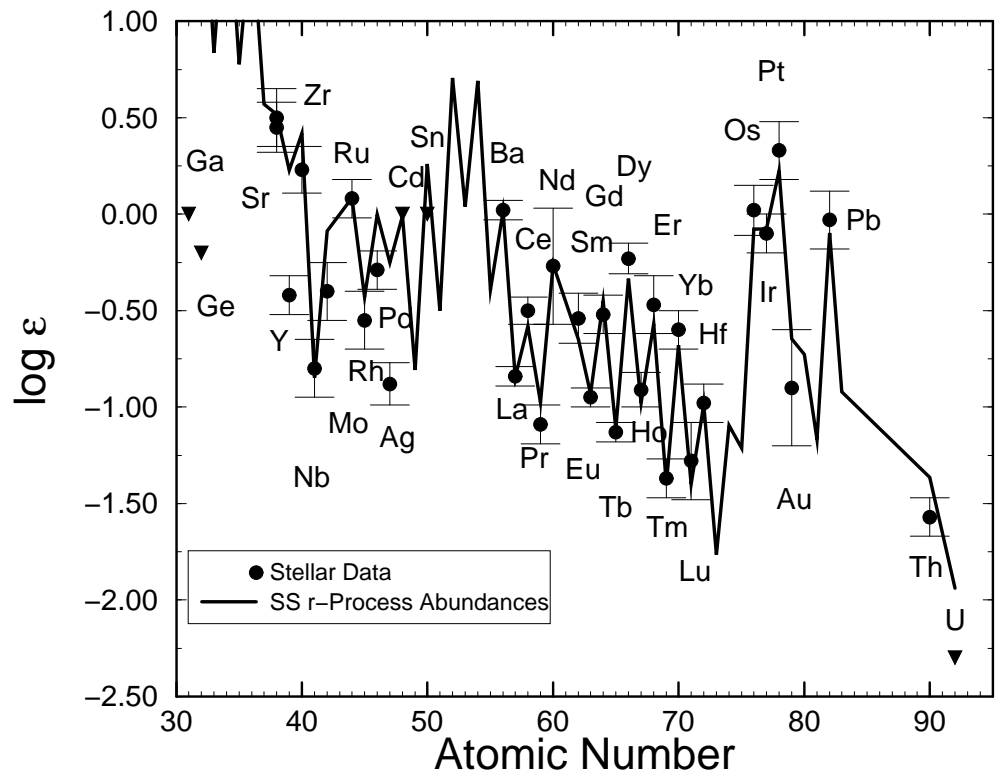

Fig. 1.2. Comparison of the observed $n$-capture abundances in CS 22892-052 from Sneden et al. (2003) and the solar system $r$-process abundance curve. Upper limits indicated by inverted triangles.

there are two sites for the $r$-process with SNe of different mass ranges and frequencies responsible for different (i.e., the heavier and lighter element) ends of the abundance distribution (Qian \& Wasserburg 2000). Some recent studies have examined the possibility of neutron-star (NS) binaries as sites for the $r$-process (Freiburghaus et al. 1999; Rosswog et al. 1999). If multiple sites are required to reproduce the heavy element abundance patterns observed in the metal-poor halo stars, then perhaps a combination of NS binaries and certain SNe might both contribute. Other studies have suggested, in contrast, that only one site, a single core-collapse supernova, could synthesize the entire observed abundance distribution (Cameron 2001).

\subsection{Abundance scatter in the early Galaxy}

Observational studies have demonstrated the abundance scatter of $n$-capture elements in the early Galaxy (Gilroy et al. 1988; Burris et al. 2000). Specifically, it is observed that there is an increasing level of star-to-star scatter of $[\mathrm{Eu} / \mathrm{Fe}]$ with decreasing metallicity (i.e., below $[\mathrm{Fe} / \mathrm{H}] \approx-2.0$ ), that is muted at higher metallicities. These results suggest an early, chemically unmixed 
and inhomogeneous Galaxy (Truran et al. 2002; Sneden \& Cowan 2003). The observed abundance scatter also strongly suggests that the bulk of the $r$-process and the iron cannot be synthesized in the same stars (Fields, Truran, \& Cowan 2002). Wheeler, Cowan, \& Hillebrandt (1998) employed the (1) Galactic abundance scatter, (2) consistency of the abundance pattern for $r$-process elements in low-metallicity halo stars, (3) monotonic evolution of low-mass cores and (4) possibility of $n$-capture synthesis either in prompt or delayed explosions to suggest that the main $r$-process site was low-mass $\left(8-10 M_{\odot}\right)$, collapsing $\mathrm{O} / \mathrm{Ne} / \mathrm{Mg}$ cores. More recent studies seem to support that conclusion that low-mass $\mathrm{SNe}$ are promising $r$-process sites (Fields et al. 2002; Wanajo et al. 2003; Ishimaru et al. 2003). While it is not possible yet to exclude high-mass SNe, recent chemical evolution models, constrained by the observed abundance scatter, do seem to rule out NS binaries as the primary $r$-process site (Argast et al. 2003).

\subsection{Summary and clues to the $r$-process}

Abundance studies have identified a number of $n$-capture elements in the metal-poor Galactic halo stars. The presence of these elements in these old stars demonstrates that $r$-process nucleosynthesis occurred early in the Galaxy. It also suggests that the $r$-process sites, the earliest stellar generations and the progenitors of the halo stars, must have been rapidly evolving and points to massive stars and SNe. The heavy $(\mathrm{Z} \geq 56) n$-capture element abundance pattern in the halo stars is consistent with the scaled solar system $r$-process distribution. This suggests a robust $r$-process operating over many Gyr and constrains the astrophysical conditions, and perhaps the supernova mass range, for the operation of the $r$-process. The abundance comparisons also demonstrate that the $n$-capture elements, including those such as $\mathrm{Ba}$ that are typically formed in the $s$-process, were predominantly synthesized by the $r$-process early in the history of the Galaxy. The lighter element $n$-capture abundances in the star CS 22892-052, in general, fall below the same solar system $r$-process abundance curve that matches the heavier $(\mathrm{Z} \geq$ 56) elemental abundances. This suggests either two separate $r$-process sites - different mass ranges and/or frequencies of supernovae or a combination of supernovae and NS-binaries - or two sets of conditions in the same supernova site. Star-to-star abundance scatter in the $n$-capture elements, such as $\mathrm{Eu}$, is increasingly constraining the possible models for early Galactic nucleosynthesis suggesting that not all supernovae are responsible for synthesizing the $r$-process. Low-mass $\mathrm{SNe}$ appear to be a promising site for the $r$-process, although high-mass objects cannot be excluded at this time. NS 
binary models do not seem consistent with this early synthesis and suggest that they are not the primary $r$-process sites. Clearly, additional observational and theoretical studies will be required to help understand better the $r$-process and identify the astrophysical sites for this process.

Acknowledgments This research has been supported in part by NSF grants AST-9986974 and AST-0307279 (JJC), AST-9987162 and AST-0307495 (CS), and by STScI grants GO-8111 and GO-08342.

\section{References}

Argast, D., Samland, M., Thielemann, F.-K., \& Qian, Y.-Z., 2003. Astron. Astrophys., in press.

Burris, D. L., Pilachowski, C. A., Armandroff, T. A., Sneden, C., Cowan, J. J., \& Roe, H., 2000. Astrophys. J., 544, $302-319$.

Busso, M., Gallino, R., \& Wasserburg, G.J., 1999. Ann. Rev. Astron. Astrophys., 37, $239-309$.

Cameron, A. G. W., 2001. Astrophys. J., 562, $456-469$.

Cowan, J. J., et al., 2002. Astrophys. J., 572, $861-879$.

Cowan, J. J., \& Sneden, C., 2003. To appear in Carnegie Observatories Astrophysics Series, Vol. 4: Origin and Evolution of the Elements, eds McWilliam, A. \& Rauch, M. Cambridge, Cambridge Univ. Press.

Fields, B. D., Truran, J. W. \& Cowan, J. J., 2002. Astrophys. J., 575, $845-854$.

Freiburghaus, C., Rosswog, S. \& Thielemann, F.-K., 1999. Astrophys. J., 525, L121 - L124.

Gilroy, K. K., Sneden, C., Pilachowski, C. A. \& Cowan, J. J., 1988. Astrophys. J., $\mathbf{3 2 7}, 298-320$.

Hill, V., et al., 2002. Astron. Astrophys., 387, $560-579$.

Ishimaru, Y., Wanjo, S., Aoki, W. \& Ryan, S. G., 2003, Astrophys. J., in press.

Johnson, J. A., \& Bolte, M., 2001. Astrophys. J., 554, 888 - 902.

McWilliam, A., Preston, G. W., Sneden, C. \& Searle, L., 1995. Astron. J., 109, $2757-2799$.

Qian, Y.-Z., \& Wasserburg, G.J. 2000, Phys. Rep., 333-334, 77 - 108.

Rosswog, S., Liebendorfer, M., Thielemann, F.-K., Davies, M. B., Benz, W., \& Piran, T., 1999. Astron. Astrophys., 341, $499-526$.

Ryan, S. G., Norris, J. E., \& Beers, T. C., 1996. Astrophys. J., 471, $254-278$.

Sneden, C., et al., 2003. Astrophys. J., 591, $936-953$.

Sneden, C. \& Cowan, J. J., 2003. Science, 299, $70-75$.

Sneden, C., McWilliam, A., Preston, G. W., Cowan, J. J., Burris, D. L. \& Armosky, B. J., 1996. Astrophys. J., 467, $819-840$.

Spite, M., \& Spite, F., 1978. Astron. Astrophys., 67, 23 - 31.

Truran, J. W., Cowan, J. J., Pilachowski, C. A. \& Sneden, C., 2002. Pub. Astron. Soc. Pac., 114, 1293 - 1308.

Wanajo, S., et al., 2003. Astrophys. J., 593, $968-979$.

Westin, J., Sneden, C., Gustaffson, B. \& Cowan, J. J., 2000. Astrophys. J., 530, $783-799$.

Wheeler, J. C., Cowan, J. J. \& Hillebrandt, W., 1998. Astrophys. J., 493, L101 L104. 